encompassing the group genetically at high risk. Detailed analysis of individual deaths during treatment does not favour any severe risk, particularly of non-accidental violent death'; complete abolition of coronary heart disease would increase life expectancy by only three years, premature mortality and morbidity being the $\mathrm{key}^{\prime}$; and a naturally low cholesterol concentration may increase the risk of stroke while reducing the risk of coronary heart disease. It is all a question of balance.

It is a proper concern that treatment may on balance be harmful in some patients, although any mechanisms are wholly speculative, but we also know that the special minority do badly without treatment. At a meeting organised by the Royal Statistical Society recently Michael Oliver was emphatic that young, middle aged, or clinically affected men with cholesterol concentrations above $7.5 \mathrm{mmol} / \mathrm{l}$ were potential candidates for lipid lowering drugs (a lower intervention poin than many propose), endorsing the thrust if not the style of his editorial. ${ }^{+} \mathrm{A}$ blanket moratorium on treatment is nonsense, but we do need to sharpen up perceptions of when and when not to introduce lipid lowering as a part of a sensible treatment plan. TONY WINDER

Department of Chemical Pathology and Human Metabolism, Roval Free Hospital School of Medicine, London NW3 2QG

1 Davey Smith G, Pekkanen J. Should there be a moratorium on cholesterol lowering drugs? B.Mf 1992:304:431-4. (15 February.)

$2 W_{\text {ysowski DK, Gross TP. Deaths due to accidents and violence }}$ in two recent trials of cholesterol-lowering drugs. Arch Interm Med 1990;150:2169-72.

3 Tserat J, Weinstein MC, Williams LW, Tosteson ANA, Goldman L. Expected gains in life expectancy from variou Goldman L. Expected gains in life expectancy from various 1991;83:1194-201.

+ Oliver MF. Doubts about preventing coronary heart disease. B.HF 1992:304:393-4. (15 February.

SIR,--George Davey Smith and Juha Pekkanen inappropriately include the expanded clinical evaluation of lovastatin (EXCEL) study ${ }^{1}$ in their meta-analysis of cholesterol lowering interventions for primary prevention of coronary disease and misinterpret it to support their argument that cholesterol lowering treatment may not reduce mortality. " The objective of the EXCEL study was to evaluate the safety of the 3-hydroxy-3methylglutaryl coenzyme A reductase inhibitor lovastatin in a large trial. It was not designed to show primary prevention: the 48 weeks of treatment was much too short for this purpose, and $28 \%$ of the patients had known ischaemic heart disease at baseline. No study of primary or seondary prevention with a 3-hydroxy-3-methylglutaryl coenzyme A reductase inhibitor has yet been completed.

The EXCEL study randomised 8245 patients into five equal groups taking lovastatin $20 \mathrm{mg}$ daily, $40 \mathrm{mg}$ daily, $20 \mathrm{mg}$ twice daily, or $40 \mathrm{mg}$ twice daily, or placebo. There were nine, five, 10 , nine, and three deaths, respectively, in the five groups. These differences did not approach significance ( $p>0 \cdot 2$ by the Kaplan-Meier method). As the authors reported, of the 36 deaths, 31 were due to coronary disease, two to a pulmonary embolism after surgery, and one each to a ruptured aortic aneurysm, a haemorrhagic stroke, and viral pneumonia. Twenty five of these 36 patients had known ischaemic heart disease at baseline.

An adverse effect of lovastatin on coronary mortality is highly improbable for several reasons. Firstly, there was a trend towards fewer nonfatal myocardial infarctions in the groups given lovastatin (10, three, 13, 11 , and 18 patients, respectively). Secondly, lovastatin when given concomitantly with a bile acid sequestrant slows the progression and induces regression of atheromatous coronary lesions. 'Thirdly, as Davey Smith and Pekkanen's meta-analysis shows, there is good evidence that lipid lowering treatment reduces deaths from coronary heart disease.

A point more relevant to the issues that Davey Smith and Pekkanen raise is that no traumatic deaths occurred in the EXCEL study. The same is true for a five year study of lovastatin in 745 patients $^{3}$ (lovastatin study groups, unpublished results); and in a one year study in 2361 patients of the closely related simvastatin one accidental death occurred. These three studies together provide roughly 12000 patient years of vigorous lipid lowering treatment with only one death due to an accident and none due to suicide or homicide. Only the lovastatin five year study is long enough to be informative on the incidence of cancer, which was well below that expected on an actuarial basis (lovastatin study groups, unpublished results). Whatever the explanation for the differences in non-cardiac mortality reported in some of the studies of older drugs, there is no evidence that lovastatin or simvastatin has any effect on non-cardiac mortality.

J A TOBERT

Merck Sharp and Dohme Research Laboratorie

PO Box 2000

Rahway, New Jersey 07065-0900,

USA

I Bradford RH, Shear CL, Chremos AN, Dujovne C, Downton M Franklin FA, et al. Expanded clinical evaluation of lovastatin (EXCEL) study results. I. Efficacy in modifying plasm lipoproteins and adverse event profile in 8245 patients with moderate hypercholesterolemia. Arch Intern Med 1991;151:43-9.

Davey Smith G, Pekkanen J. Should there be a moratorium on the use of cholesterol lowering drugs? BMF 1992;304:431-4 (15 February.)

3 Brown G, Albers JJ, Fisher LD, Schaefer FM, Lin J, Kaplan C, et al. Regression of coronary artery disease as a result of et al. Regression of coronary artery disease as a result of
intensive lipid-lowering therapy in men with high levels of intensive lipid-lowering therapy in men with hig
apolipoprotein B. N Engl F Med 1990;323:1289-98.

+ Tobert JA. Cholesterol lowering and non-cardiac mortality. Lancet 1991;338:126.

5 Boccuzzi SJ, Bocanegra TS, Walker JF, Shapiro DR, Keegan ME. Long-term safety and efficacy profile of simvastatin Am f Cardiol 1991;68:1127-31.

\section{Managing hypertension}

SIR,-We were interested in the results of the Medical Research Council's trial of treatment of hypertension in older adults and their implications for the treatment of hypertensive patients of all ages, particularly the prevention of stroke. Individual studies and meta-analyses show that about $40 \%$ of stroke in hypertensive patients can be prevented by drug treatment. ${ }^{2}$

In the Western Infirmary we recently established an acute stroke unit, which admits around 300 unselected patients each year from a catchment population of 220000 . Blood pressure immediately after stroke may increase temporarily. We have information on previous diagnosis and treatment for 351 patients (159 men (median age 67) and 192 women (median age 74$)$ ). Of these, 117 were aged $70-79$ and 80 were aged $\geqslant 80$. Hypertension had been diagnosed in 132 patients (information obtained from the patient, relatives, or general practitioner's referral letter), but only 62 were receiving drug treatment: $12(44 \%)$ of the 27 aged $\geqslant 80,23(49 \%)$ of the 47 aged $70-79$, and 27 $(47 \%)$ of the 58 aged $<70$. Thirty seven $(60 \%)$ of the 62 receiving treatment for hypertension had at least one other major cardiovascular risk factor, as did $30(43 \%)$ of the 70 not receiving treatment.

We do not know why antihypertensive treatment was not offered to or continued in patients at high risk of stroke and vascular disease. Some of the patients had been treated previously but had either stopped taking their drugs or had their treatment stopped weeks or months before presenting with stroke. Despite the clear evidence of the value of antihypertensive treatment in younger patients that has been available for many years it is surpris ing that the "rule of halves" still holds: up to half of all hypertension is unrecognised and only half of detected hypertension is treated. It remains to be seen whether further evidence of the benefits of treating elderly hypertensive patients will influence medical practice. If it does not many strokes that are preventable will occur, with resultant disability and death.

\section{KENNEDY R LEES GORDON T MCINNES}

JOHN L REID

IAIN B SQUIRE

Department of Medicine and Therapeutics,

University of Glasgow,

Gardiner Institute,

Glasgow Gil 6NT

1 MRC Working Party. Medical Research Council trial of treatment of hypertension in older adults: principal results. $B M \mathcal{Y}$ 1992;304:405-12. (15 February.)

2 Beard K, Bulpitt C, Mascie-Taylor H, O'Malley K, Severe P, Webb S. Management of elderly patients with sustained Webb S. Management of elderly patients with
hypertension. BMf 1992;304:412-6. (15 February.)

3 Collins R, Peto R, MacMahon S, Hebert P, Fiebach NH, Eberlein KA, et al. Blood pressure, stroke, and coronary hear disease. Part 2. Short term reductions in blood pressure: overview of randomised drug trials in their epidemiologica context. Lancet 1990;335:827-38.

\section{Thrombosis and pulmonary embolism}

SIR, - In their article on thrombosis and pulmonary embolism N F G Hopkins and John H N Wolfe describe chest radiographic findings in pulmonary embolism without making it clear that, in most cases, there is either no abnormality or minimal, non-specific change in the plain chest film.

In their discussion of ventilation-perfusion lung scans they do not mention that scans are classified as showing a normal or very low, a low, an intermediate or indeterminate, or a high probability of embolism. Though the first and last of these groups can be taken at face value (although even they are not $100 \%$ guarantees), patients with scans showing a low probability were shown by the prospective investigation of pulmonary embolism diagnosis to have a $14 \%$ incidence of embolism. ${ }^{2}$ Either one has to do pulmonary arteriography on, at least, all patients with scans showing an indeterminate probability or one adopts the policy that in the absence of a scan showing a high probability and of persisting venous thrombosis (diagnosed by whatever means) pulmonary embolism, even if present, does not require treatment. One cannot, however, just take ventilationperfusion scanning as a test that will in every case tell for certain whether pulmonary embolism is present.

With respect to the investigation of deep venous thrombosis, as stated venography remains the standard test. Ultrasound can be used in severa ways. Duplex Doppler ultrasonography will show venous patency accurately from the poplitea vein up, and the use of colour flow even allows assessment in the calf and will show non-occlusive thrombus. Without Doppler, however, ultrasound relies on the compression method. At best this is of use only in the groin and upper thigh and in the popliteal fossa (the vein being too deep in the adductor canal), and even in the groin noncompressibility can be normal where the long saphenous vein enters the femoral vein.

Radioisotope studies such as those using labelled fibrinogen or plasmin show only forming thrombus, not that already present. Hence anticoagulant treatment cannot be started until imaging is complete. Labelled antifibrin monoclonal antibodies will, however, target formed thrombus. Another method, not referred to in the article, is light reflective rheography. Though this is nonspecific (a positive result indicating venous abnormality but not necessarily thrombus), it is highly sensitive and could serve as a screening test to 
prevent a patient who definitely has no abnormality from proceeding to venography. ${ }^{+}$

V C WILLIAMSON

Department of Diagnostic Radiology,

Walton Hospital,

Liverpool L9 $1 \mathrm{AE}$

P CANTRELL

Department of Diagnostic Radiology,

Royal Liverpool Hospital,

Liverpool L7 8XP

1 Hopkins NFG, Wolfe JHN. Thrombosis and pulmonary embolism. BMF 1991;303:1260-2. (16 November.)

2 PIOPED Investigators. The value of the ventilation/perfusion scan in acute pulmonary embolism; results of the prospective investigation of pulmonary embolism diagnosis. JAMA 1990;263:2753-9.

3 Sissons GRJ, Pugh ND. Venous ultrasound in the thigh: norma variants and potential pitfalls. Brf Radiol 1991;64(suppl):27.

4 Harris I, Diggory RS, Abbot GT. Light reflective rheography as a firt line in thrombosis. Br f Radiol 1991;64(suppl): 57 .

SIR, - N F G Hopkins and John H N Wolfe's article on thrombosis and pulmonary embolism in the ABC of Vascular Diseases contained several errors.

Firstly, the British Committee for Standards in Haematology, Haemostasis and Thrombosis Task Force of the British Society for Haematology suggests that after a baseline prothrombin time has been determined the usual induction dose of warfarin in adults should be $10 \mathrm{mg}$ on the first day and $10 \mathrm{mg}$ on the second day. ${ }^{2}$ The large induction dose of $20-30 \mathrm{mg}$ suggested in the article is no longer recommended, especially as there are theoretical concerns that such an approach may induce a transient hypercoaguable state due to rapid reductions in the vitamin $K$ dependent anticoagulants, protein $C$ and protein $S$.

Secondly, the same body now recommends that treatment with warfarin should be aimed at keeping the prothrombin time at between two and three times the normal value, using international reference thromboplastin, in deep vein thrombosis and pulmonary embolism. ${ }^{2}$ The lower value of 1.7 stated in the article is thus misleading.

Thirdly, after heparinisation the presently recommended value for the activated partial thromboplastin time is $1 \cdot 5-2 \cdot 0$ times the upper limit of control values. ${ }^{+}$Values 2-3 times control values are associated with significantly more bleeding complications and have no therapeutic benefits over the lower value. ${ }^{5}$

Fourthly, the article gives correct recommendations for conservative treatment of deep vein thrombosis in the calf but should also state that this is acceptable only if extension of the clot is excluded by repeated monitoring by ultrasonography, as $20-30 \%$ of thrombi in calf veins extend into the proximal veins.

Finally, the illustration of the ischaemic limb induced by heparin states that this is due to thrombocythaemia. This should presumably read heparin induced thrombocytopenia.

A M ROBINSON

Professorial Medical Unit,

Royal Hallamshire Hospital,

Sheffield S10 2JF

1 Hopkins NFG, Wolfe JHN. Thrombosis and pulmonary embolism. BM7 1991:303:1260-2. (16 November.)

2 British Committee for Standards in Haematology, Haemostasis and Thrombosis Task Force of the British Society for Haem tology. Guidelines on oral anticoagulation: second edition. f Clin Pathol 1990;43:177-83.

3 Greaves M, Preston FE. The hypercoaguable state in clinical practice. Brf Haematol 1991;79:148-51

4 Hirsh J. Heparin. N Engl f Med 1991;324:1565-74.

5 Hirsh J. Antithrombotic therapy. In: Hirsh J, ed. Clinical haematology. Vol 3, No 3. London: Baillière Tindall, 1990: 686-7.

6 Kakker VV, Flac C, Howe CT; Clarke MB. Natural history of post-operative vein thrombosis. Lancet 1969;ii:230-2.

AUTHORS' REPLY,-These comments on our article are well taken, but I am sure that the authors appreciate that an essential aspect of articles in
ABC series is their brevity and simplicity. We therefore pointed out the abnormalities that migh be evident in chest $x$ ray films and ventilationperfusion scans without implying that these are always present. We made it clear that fibrinogen labelled with iodine-125 is useful only in a screening test and is not, therefore, part of the norma armamentarium in diagnosing deep vein throm bosis. Though labelled antifibrin monoclonal antibodies are of great interest to specialists, they are not yet widely available.

We are glad that the authors agree with our point about ultrasound but would point out that it is the Doppler probe that relies on the compression method and it is the ultrasound modality that is present in the duplex Doppler system.

We are glad of A M Robinson's comments about the dose of warfarin as we should have emphasised that the loading dose was $20-30 \mathrm{mg}$ over three days and then daily, which is similar to the new recommendations of the British Committee for Standards in Haematology, Haemostasis, and Thrombosis. Again, the new suggested values for prothrombin time-2-3 times the normal valuevary only little from our suggestion of $1 \cdot 7-3 \cdot 0$.

We entirely agree that calf vein thrombi should be treated conservatively only if they are not subsequently shown to extend into the popliteal and superficial femoral veins, but the essential point is that patients with popliteal thrombi should be mobilised and should not be admitted to hospital or treated.

Regional Vascular Unit,

St Mary's Hospital,

London W2 INY

J H N WOLFE

Crawley Hospital

Crawley,

West Sussex RH11 7DH

\section{Age associated memory impairment}

SIR,-John T O'Brien and Raymond Levy observed that "cognitive function fails with age." They went on to consider clinical classification and treatment of older people who have experienced loss of memory and other cognitive abilities but who are not demented. The authors contrasted the diagnostic criteria for "benign senescen forgetfulness" proposed by Kral several decades ago $^{2}$ with those for "age-associated memory impairment" proposed by a National Institute of Mental Health work group that we had the privilege to cochair in $1985 .^{3}$ O'Brien and Levy pointed out that the principal difference between the two approaches is that the Kral criteria describe people whose memory performance is impaired relative to that of other people of the same age, while the National Institute of Mental Health criteria describe people who report that their memory has declined since they were young adults and who perform at a level significantly lower than that of young adults. In discussing the development of treatments for memory loss in later life O'Brien and Levy argued that the National Institute of Mental Health criteria are too broad to be usefu and that, as Kral proposed, memory impairmen should be redefined using age standardised norma values, rather than those of young adults.

In considering O'Brien and Levy's argumen one might draw a parallel with other conditions in which prevalence is increased in later life. For example, presbyopia is defined by reference to normative standards for young adults and is so common among the elderly as to be considered "normal." Nevertheless, few clinicians would compare the vision of an 80 year old with norms established for other people of the same age and prescribe corrective lenses only to those whose visual performance falls outside those norms. As in the case of vision, considerable changes in memory may occur with advancing age among healthy "normal" people. Deficits of $50 \%$ and more may be expected between age 25 and age 75 in the ability to perform such important everyday memory tasks as learning and remembering names of people to whom one is introduced or learning new verbal information. ${ }^{45} \mathrm{O}$ 'Brien and Levy described such problems as "just a benign inconvenience of growing old," but one might ask whether a cognitive deficit of $50 \%$ would be considered "benign" if seen in a young adult.

A well developed body of preclinical data ${ }^{6-8}$ and emerging clinical evidence ${ }^{90}$ suggest that memory deficits associated with normal aging-that is, age associated memory impairment-may be diminished through pharmacological treatment. The argument of O'Brien and Levy that this possibility should not even be investigated is difficult to justify scientifically and may be difficult to justify to the many older patients who might benefit.

THOMAS H CROOK

Advanced Psychometrics Corporation

Bethesda

Maryland 20814

USA

epartment of Psychiatry,

New York University School of Medicine,

New York,

New York 10016,

USA

1 O'Brien JT, Levy R. Age associated memory impairment. BMY 1992;304:5-6. (4 January.)

$2 \mathrm{Kral}$ VA. Senescent forgetfulness: benign and malignant. $\mathrm{Can}$ Med Assoc f 1962;86:257-60.

3 Cook TH, Bartus RT, Ferris SH, Whitehouse P, Cohen GD, Gershon S. Age-associated memory impairment: proposed diagnostic criteria and measures of clinical change. Repor of a National Institute of Mental Health Work Group. Developmental Neuropsychology 1986:2:261-76.

4 Crook TH, West RL. Name recall performance across the adult life span. Br f Psychology 1990;81:335-49.

5 Youngiohn JR, Larrabee GJ, Crook TH. First-last names and the grocery list selective reminding tests: two computerized measures of everyday verbal learning. Arch Clin Neuropsychol 991;6:287-300

6 McEntee WJ, Crook TH. Age-associated memory impairment: a role for catecholamines. Neurology 1990;40:526-30

7 McEntee WJ, Crook TH. Serotonin, memory, and the agin brain. Psychopharmacology 1991;103:143-9.

8 Bartus RT, Dean RL, Beer B, Lippa AS. The cholinergic hypothesis of geriatric memory. Science 1902;217:408-17.

9 Crook T, Tinklenberg J, Yesavage J, Petrie W, Nunzi MG, Massari D. Effects of phosphatidylserine in age-associated memory impairment. Neurology 1991;41:644-9.

10 Crook T, Lakin $M$. Effects of ondansetron in age-associated memory impairment. In: Racagni G, ed. Biological psychiatry. Vol 2. Amsterdam: Elsevier, 1991:888-90.

\section{Mortality in meningococcal disease}

SIR,-Keith Cartwright and colleagues recommend giving benzylpenicillin parenterally when meningococcal disease is suspected. ${ }^{1}$ Their laudable aim is to reduce the case mortality of meningococcal disease by giving an effective antimicrobia drug at an early stage. They rightly point out that the initial rash in meningococcal disease may not be a "typical" petechial rash. Some patients do not show a rash of any type at any stage of their illness.

Because an effective antimicrobial drug such as benzylpenicillin almost invariably clears the blood stream of meningococci rapidly, blood should be cultured before the first dose of benzylpenicillin is given. General practitioners should therefore consider carrying a blood culture kit in their emergency bag.

In South Australia we are faced with similar problems in bringing patients with suspected meningococcal disease to hospital, sometimes from considerable distances. The resuscitation team takes a blood culture before chemotherapy is started. In children systemic infection with other encapsulate bacteria, especially Haemophilus influenzae type $\mathrm{b}$ and pneumococci, may simulate 